\title{
Safety of Proton-Pump Inhibitors in High-Risk Cardiovascular \\ Subsets of the COGENT Trial
}

Muthiah Vaduganathan, MD MPH${ }^{1}$; Christopher P. Cannon, MD ${ }^{1,2}$; Byron L. Cryer, MD; Yuyin Liu, MS ${ }^{2,4}$; Wen-Hua Hsieh, PhD $^{2}$; Gheorghe Doros, $\mathrm{PhD}^{2,4}$; Marc Cohen, MD; Angel Lanas, MD DSc ${ }^{6}$; Thomas J. Schnitzer, MD PhD; Thomas L. Shook, MD; Pablo Lapuerta, MD ${ }^{9}$; Mark A. Goldsmith, MD PhD ${ }^{10}$; Loren Laine, MD ${ }^{11}$; Deepak L. Bhatt, MD $\mathrm{MPH}^{1}$; on behalf of the COGENT Investigators*

${ }^{1}$ Brigham and Women's Hospital Heart \& Vascular Center and Harvard Medical School, Boston, MA, USA

${ }^{2}$ Harvard Clinical Research Institute, Boston, MA, USA

${ }^{3}$ University of Texas Southwestern and Veterans Affairs North Texas Health Care System, Dallas, TX, USA

${ }^{4}$ Department of Biostatistics, Boston University, Boston, MA, USA

${ }^{5}$ Newark Beth Israel Medical Center, Newark, NJ, USA

${ }^{6}$ University of Zaragoza, Instituto de Investigación Sanitaria Aragón, Centro de Investigación Biomédica en Red en Enfermedades Hepáticas y Digestivas, Zaragoza, Spain

${ }^{7}$ Departments of Physical Medicine and Rehabilitation and Internal MedicineRheumatology, Northwestern University Feinberg School of Medicine, Chicago, IL, USA ${ }^{8}$ PAREXEL Parexel-International, Waltham, MA, USA

${ }^{9}$ Lexicon Pharmaceuticals, Princeton, NJ, USABasking Ridge, NJ, USA 
${ }^{10}$ Constellation Pharmaceuticals, Cambridge, MA, USA

${ }^{11}$ Yale School of Medicine, New Haven, CT and VA Connecticut Healthcare System, West Haven, CT, USA

*A full list of investigators can be found in Bhatt DL et al. N Engl J Med 2010;363:19091917.

Authorship Statement: All authors had access to the data and a role in writing the manuscript

Article Type: Brief Observation

Key Words: acute coronary syndrome; bleeding; clinical outcomes; clinical trials; coronary artery disease; percutaneous coronary intervention; proton-pump inhibitors Running Head: Safety of PPIs in High-Risk Cardiovascular Subsets after ACS or PCI

Word Count: $1,716 \underline{1,886}$

Address for Correspondence: Deepak L. Bhatt, MD, MPH, FACC, FAHA, FACP; Brigham and Women's Hospital Heart \& Vascular Center, 75 Francis St, Boston, MA 02115; Tel 857-307-1992; Fax 857-307-1955; Email dlbhattmd@post.harvard.edu 


\section{STRUCTURED ABSTRACT}

Background: Proton-pump inhibitors (PPIs) have been demonstrated to reduce rates of gastrointestinal (GI) events in patients requiring dual antiplatelet therapy (DAPT). Data are limited regarding the efficacy and safety of PPIs in high-risk cardiovascular subsets after acute coronary syndrome (ACS) or percutaneous coronary intervention. $(\mathrm{PCI})$. Methods: These post hoc analyses of the COGENT (Clopidogrel and the Optimization of Gastrointestinal Events Trial) trial evaluated the efficacy and safety of omeprazole compared with placebo in All patients enrolled in COGENT (Clopidogrel and the Optimization of Gastrointestinal Events Trial) were initiated on DAPT (with aspirin and clopidogrel) for various indications within the prior 21 days. These post hoc analyses of the COGENT trial evaluated the efficacy and safety of omeprazole compared with placebo in subsets of patients requiring DAPT for the two most frequent indications: 1) 1) patients undergoing PClpercutaneous coronary intervention (for any indication) within 14 days of randomization-and receiving DAPF ( $n=2,676 ; 71.2 \%)$; and 2$)$ patients

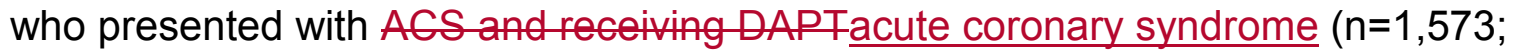
41.8\%). Unadjusted Cox proportional hazards models were used to estimate effect sizes through final follow-up.

Results: Median follow-up duration was 110 days (IQR 55 to 167). In PClpercutaneous coronary intervention-treated patients, omeprazole significantly reduced rates of composite Glgastrointestinal events at 180 days $(1.2 \%$ vs. $2.7 \% ; \mathrm{HR} 0.43,95 \% \mathrm{Cl}$ $0.22-0.85 ; p=0.02)$ without increasing composite cardiovascular events $(5.4 \%$ vs. $6.3 \%$; HR $1.00,95 \% \mathrm{Cl} 0.67-1.50 ; p=1.00)$. Similarly, omeprazole lowered risk of the primary 
gastrointestinal Glendpoint at 180 days in patients presenting with acute coronary syndrome ACS-(1.1\% vs. $2.7 \%$; HR 0.37, 95\% Cl 0.13-1.01; $\mathrm{p}=0.05)$ without a significant excess in cardiovascular events (5.6\% vs. 4.5\%; HR 1.40, 95\% Cl 0.77-2.53; $p=0.27)$.

Conclusions: PPI therapy attenuates gastrointestinal GHbleeding risk without a significant excess in major cardiovascular events in high-risk cardiovascular subsets, regardless of indication for DAPT. Future studies will be needed to clarify optimal gastroprotective strategies for higher-intensity and longer durations of DAPT. 
Major gastrointestinal(GI) bleeding after acute coronary syndromes(ACS) or in patients undergoing percutaneous coronary intervention $(\mathrm{PCl})$ is common and is associated with adverse prognosis. ${ }^{1}$ The COGENT (Clopidogrel and the Optimization of Gastrointestinal Events Trial; ClinicalTrials.gov Identifier NCT00557921) trial demonstrated that omeprazole reduced rates of composite gastrointestinal $G+$ events at 180 days $^{2}$ and patient-reported dyspepsia ${ }^{3}$ compared with placebo in patients with coronary artery disease $(C A D)$ requiring $\geq 12$ months of dual antiplatelet therapy (DAPT) for any indication without adversely influencing risk of major adverse cardiovascular events. Despite these data, safety concerns persist regarding the generalizability of this randomized experience to high-risk patients after acute coronary syndrome ACS or

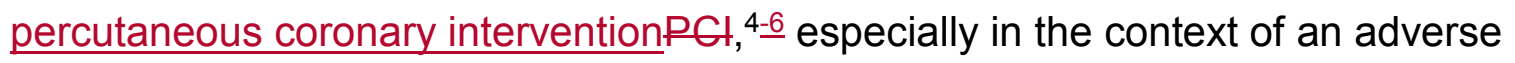
pharmacodynamic interaction between proton-pump inhibitors (PPIs) and clopidogrel. ${ }^{75}$ ${ }^{108}$ Furthermore, although PPIs are often administered to hospitalized patients presenting with acute coronary syndrome ACS-or for percutaneous coronary intervention PCI, continuation of PPI therapy post-discharge is a question faced by many outpatient clinicians. As such, we report the efficacy and safety of PPI therapy in highrisk, enriched subgroups after acute coronary syndrome ACS-or percutaneous coronary intervention $\mathrm{PCH}$ in the COGENT trial. 


\section{METHODS}

As previously described, ${ }^{2}$ COGENT was a phase-3, multicenter, global, placebocontrolled, double-blind, double-dummy randomized controlled trial of a fixedcombination of clopidogrel $75 \mathrm{mg}$ and omeprazole $20 \mathrm{mg}$ compared with clopidogrel $75 \mathrm{mg}$ alone. Enteric-coated aspirin was provided to all study patients. Patients initiated on DAPT within the prior 21 days without use of recent gastroprotection, oral anticoagulation, or fibrinolytic therapy were eligible for enrollment. The ethics committees and institutional review boards of each individual site locally approved the study protocol and all patients provided explicit informed consent for trial participation. The primary adjudicated composite gastrointestinal Glendpoint included overt upper gastrointestinal GH-bleeding, bleeding of presumed gastrointestinal GHorigin, symptomatic gastroduodenal ulcer, endoscopy-confirmed gastroduodenal erosions, obstruction, or perforation. The secondary adjudicated gastrointestinal Glendpoint for the present analysis was overt upper gastrointestinal GHbleeding (known or unknown origin). The primary adjudicated cardiovascular endpoint was the composite of cardiovascular death, non-fatal myocardial infarction, coronary revascularization, or ischemic stroke.

Number of patients who experienced events on or before 180 days and KaplanMeier estimates of event rates at 180 days are presented for patients with or without acute coronary syndrome ACS-and with or without percutaneous coronary interventionPCI. Interaction analyses between treatment assignment (with PPI or placebo) and DAPT indication were performed using Breslow-Day tests. Effect sizes through final follow-up were estimated using unadjusted Cox proportional hazards 
models, expressed as hazard ratios (HR) and 95\% confidence intervals $(\mathrm{Cl})$. All statistical analyses were performed using SAS version 9.4 (SAS Institute Inc., Cary, $\mathrm{NC})$.

\section{RESULTS}

COGENT was terminated early due to the sponsor filing for bankruptcy. In the final intention-to-treat population $(n=3,759)$, risks of gastrointestinal Gtand cardiovascular events were assessed in two non-mutually exclusive groups (the two most common indications for DAPT): 1) patients undergoing percutaneous coronary intervention $\mathrm{PCF}$ within 14 days of randomization $(n=2,676 ; 71.2 \%)$ and 2$)$ patients who presented with acute coronary syndrome ACS- $(n=1,573 ; 41.8 \%)$. Data regarding percutaneous coronary intervention $\mathrm{PCl}$ and acute coronary syndrome ACS-status were missing in 36 and 38 patients, respectively. There were no major differences in baseline characteristics in patients randomized to omeprazole or placebo in either major subgroup (data not shown). As such, since the original randomization was preserved, no additional statistical adjustment was applied to these analyses. Despite differences in indications of DAPT utilization, baseline characteristics in the PPI and placebo groups were well-balanced across these subgroups. Median follow-up duration was 110 days (IQR 55 to 167). In percutaneous coronary interventionPCI-treated patients, omeprazole

significantly reduced rates of composite gastrointestinal Glevents $(1.2 \%$ vs. $2.7 \%$; HR $0.43,95 \% \mathrm{Cl} 0.22-0.85 ; \mathrm{p}=0.02$ ) without increasing composite cardiovascular events (5.4\% vs. $6.3 \%$; HR 1.00, 95\% Cl 0.67-1.50; $p=1.00$ ). Omeprazole lowered risk of the primary gastrointestinal Glevent in patients presenting with acute coronary syndrome 
ACS-(1.1\% vs. $2.7 \%$; HR 0.37, 95\% Cl 0.13-1.01; $p=0.05)$ without a significant excess in cardiovascular events (5.6\% vs. $4.5 \%$; HR 1.40, 95\% Cl 0.77-2.53; $p=0.27)$. Similar trends were observed for the secondary gastrointestinal G+endpoint, overt upper gastrointestinal GLbleeding (Table).

\section{DISCUSSION}

COGENT enrolled a high-risk cardiovascular cohort with over $70 \%$ of patients having undergone percutaneous coronary intervention $\mathrm{PCl}$ and over $40 \%$ presenting with acute coronary syndromeACS. Approximately $2-3 \%$ of patients experienced major gastrointestinal Glevents during 6-months of DAPT after acute coronary syndrome ACS-or percutaneous coronary interventionPCI. Consistent with the overall trial results, ${ }^{2}$ this post hoc analysis demonstrates that PPI therapy safely attenuates this gastrointestinal GI risk without significant excess in major cardiovascular events at 180 days, regardless of initial indication for DAPT. The gastroprotective efficacy of PPIs in the post-acute coronary syndromeACS setting has recently been corroborated by realworld data from a large, nationwide cohort study. $\underline{119}$

This study is subject to a number of limitations. The trial was prematurely terminated due to loss of funding prior to meeting target enrollment. However, sufficient follow-up was completed during the high-risk period after acute coronary syndrome ACS and percutaneous coronary interventionPCl. Our analyses did not account for multiple comparisons. The trial utilized a combination formulation of clopidogrel and omeprazole that is not commercially available, though it is unlikely this would affect the results. COGENT was significantly underpowered to detect differences in cardiovascular events 
in individual subgroups, and some heterogeneity around the overall trial point estimate in cardiovascular risk was expected across the tested subsets. Future studies, including COMPASS (Cardiovascular Outcomes for People Using Anticoagulation Strategies; ClinicalTrials.gov Identifier NCT01776424), will shed further light on optimal gastroprotective strategies in high-risk CAD-coronary artery disease patients requiring more potent antithrombotic combinations used for longer durations. ${ }^{12} 0$

Despite high post-acute coronary syndromeACS and post-percutaneous coronary intervention $\mathrm{PCH}$-bleeding risk on contemporary DAPT regimens, PPI use remains suboptimal in appropriately-selected patients, ${ }^{134}$ perhaps related to underestimation of gastrointestinal GLbleeding risks ${ }^{142}$ or to concerns regarding a potentially adverse drug interaction between PPIs and clopidogrel. $45-108$ These data from COGENT, the only large, randomized placebo-controlled trial evaluating effects of PPIs on clinical endpoints in patients requiring DAPT, provide reassurance regarding the safety of PPIs in high-risk cardiovascular subsets. At this juncture, use of prophylactic PPIs appears to be safe and represents a recommended strategy ${ }^{153-1 \underline{5}}$ for attenuating gastrointestinal GH-bleeding risk in patients requiring DAPT, including after percutaneous coronary intervention $\mathrm{PCI}$ or acute coronary syndromeACS.

\section{ACKNOWLEDGEMENTS}

None

\section{FUNDING}


The COGENT trial was previously-funded by Cogentus Pharmaceuticals, however this post hoc analysis was conducted independently with biostatistical support from an independent team from Harvard Clinical Research Institute (HCRI). The study investigators had full access to the trial database and retained complete control on the decision to pursue publication. The sponsor did not have right to review or approve the final manuscript.

\section{CONFLICTS OF INTEREST}

Dr. Muthiah Vaduganathan has no relevant disclosures.

Dr. Christopher P. Cannon has served on advisory boards of Bristol-Myers Squibb, Lipimedix, and Pfizer; and has received research funding from Accumetrics, Arisaph, AstraZeneca, Boehringer-Ingelheim, CSL Behring, Essentialis, GlaxoSmithKline, Janssen, Merck Regeneron, Sanofi, and Takeda.

Dr. Byron L. Cryer has served as a consultant to Cogent Pharmaceuticals.

Ms. Yuyin Liu has no relevant disclosures.

Dr. Wen-Hua Hsieh has no relevant disclosures.

Dr. Gheorghe Doros has no relevant disclosures.

Dr. Marc Cohen has no relevant disclosures.

Dr. Angel Lanas has received an investigator-initiated grant from Bayer Pharma AG and has served on advisory boards for Bayer Pharma AG.

Dr. Thomas J. Schnitzer has no relevant disclosures.

Dr. Thomas L. Shook is an employee of PAREXEL International.

Dr. Pablo Lapuerta is an employee of Lexicon Pharmaceuticals. 
Dr. Mark A. Goldsmith is an employee of Constellation Pharmaceuticals.

Dr. Loren Laine has served on the Data Safety Monitoring Boards for studies sponsored by Bayer and Bristol-Myers Squibb.

Dr. Deepak L. Bhatt discloses the following relationships - Advisory Board: Cardax, Elsevier Practice Update Cardiology, Medscape Cardiology, Regado Biosciences;

Board of Directors: Boston VA Research Institute, Society of Cardiovascular Patient Care; Chair: American Heart Association Quality Oversight Committee; Data Monitoring Committees: Duke Clinical Research Institute, Harvard Clinical Research Institute, Mayo Clinic, Population Health Research Institute; Honoraria: American College of Cardiology (Senior Associate Editor, Clinical Trials and News, ACC.org), Belvoir Publications (Editor in Chief, Harvard Heart Letter), Duke Clinical Research Institute (clinical trial steering committees), Harvard Clinical Research Institute (clinical trial steering committee), HMP Communications (Editor in Chief, Journal of Invasive Cardiology), Journal of the American College of Cardiology (Guest Editor; Associate Editor), Population Health Research Institute (clinical trial steering committee), Slack Publications (Chief Medical Editor, Cardiology Today's Intervention), Society of Cardiovascular Patient Care (Secretary/Treasurer), WebMD (CME steering committees); Other: Clinical Cardiology (Deputy Editor), NCDR-ACTION Registry Steering Committee (Vice-Chair), VA CART Research and Publications Committee (Chair); Research Funding: Amarin, AstraZeneca, Bristol-Myers Squibb, Eisai, Ethicon, Forest Laboratories, Ischemix, Medtronic, Pfizer, Roche, Sanofi Aventis, The Medicines Company; Royalties: Elsevier (Editor, Cardiovascular Intervention: A Companion to Braunwald's Heart Disease); Site Co-Investigator: Biotronik, Boston Scientific, St. Jude 
Medical; Trustee: American College of Cardiology; Unfunded Research: Cogentus

(Chair of COGENT), FlowCo, PLx Pharma, Takeda.

\section{REFERENCES}

1. Eikelboom JW, Mehta SR, Anand SS, Xie C, Fox KA, Yusuf S. Adverse impact of bleeding on prognosis in patients with acute coronary syndromes. Circulation 2006;114:774-782.

2. Bhatt DL, Cryer BL, Contant CF, et al. Clopidogrel with or without omeprazole in coronary artery disease. N Engl J Med 2010;363:1909-1917.

3. Vardi M, Cryer BL, Cohen M, et al. The effects of proton pump inhibition on patientreported severity of dyspepsia when receiving dual anti-platelet therapy with clopidogrel and low-dose aspirin: analysis from the Clopidogrel and the Optimization of Gastrointestinal Events Trial. Aliment Pharmacol Ther 2015;42:365-374.

4. Southworth MR, Temple R. Interaction of clopidogrel and omeprazole. N Engl J Med 2010;363:1977.

5. Shah NH, LePendu P, Bauer-Mehren A, et al. Proton pump inhibitor usage and the risk of myocardial infarction in the general population. PLOS One 2015;10:e0124653. 6. Sherwood MW, Melloni C, Jones WS, Washam JB, Hasselblad V, Dolor RJ. Individual proton pump inhibitors and outcomes in patients with coronary artery disease on dual antiplatelet therapy: A systematic review. J Am Heart Assoc 2015;4:e002245. 
75. Frelinger AL, 3rd, Lee RD, Mulford DJ, et al. A randomized, 2-period, crossover design study to assess the effects of dexlansoprazole, lansoprazole, esomeprazole, and omeprazole on the steady-state pharmacokinetics and pharmacodynamics of clopidogrel in healthy volunteers. J Am Coll Cardiol 2012;59:1304-1311.

86. Frelinger AL, 3rd, Bhatt DL, Lee RD, et al. Clopidogrel pharmacokinetics and pharmacodynamics vary widely despite exclusion or control of polymorphisms (CYP2C19, ABCB1, PON1), noncompliance, diet, smoking, co-medications (including proton pump inhibitors), and pre-existent variability in platelet function. J Am Coll Cardiol 2013;61:872-879.

97. Banerjee S, Weideman RA, Weideman MW, et al. Effect of concomitant use of clopidogrel and proton pump inhibitors after percutaneous coronary intervention. Am J Cardiol 2011;107:871-878.

108. Depta JP, Bhatt DL. Omeprazole and clopidogrel: should clinicians be worried? Cleve Clin J Med 2010;77:113-116.

119. Olsen AS, Lindhardsen J, Gislason GH, et al. Impact of proton pump inhibitor treatment on gastrointestinal bleeding associated with non-steroidal anti-inflammatory drug use among post-myocardial infarction patients taking antithrombotics: nationwide study. BMJ 2015;351:h5096.

120. Vaduganathan M, Bhatt DL. Revisiting the Clopidogrel-Proton Pump Inhibitor Interaction: From Bench to Bedside. Circ Cardiovasc Interv 2015;8.

13⒈ de Jong HJ, Korevaar JC, van Dijk L, Voogd E, van Dijk CE, van Oijen MG. Suboptimal prescribing of proton-pump inhibitors in low-dose aspirin users: a cohort study in primary care. BMJ Open 2013;3. 
142. Vaduganathan $\mathrm{M}$, Bhatt $\mathrm{DL}$, Cryer BL, et al. Proton-pump inhibitors reduce risk of gastrointestinal events regardless of aspirin dose in patients requiring dual antiplatelet therapy: Insights from the COGENT trial. J Am Coll Cardiol 2016 (in press).

153. Bhatt DL, Scheiman J, Abraham NS, et al. ACCF/ACG/AHA 2008 expert consensus document on reducing the gastrointestinal risks of antiplatelet therapy and NSAID use: a report of the American College of Cardiology Foundation Task Force on Clinical Expert Consensus Documents. Circulation 2008;118:1894-1909.

164. Abraham NS, Hlatky MA, Antman EM, et al. ACCF/ACG/AHA 2010 Expert Consensus Document on the concomitant use of proton pump inhibitors and thienopyridines: a focused update of the ACCF/ACG/AHA 2008 expert consensus document on reducing the gastrointestinal risks of antiplatelet therapy and NSAID use: a report of the American College of Cardiology Foundation Task Force on Expert Consensus Documents. Circulation 2010;122:2619-2633.

175. Moukarbel GV, Bhatt DL. Antiplatelet therapy and proton pump inhibition: clinician update. Circulation 2012;125:375-380. 